\title{
Dynamical Chirally Improved Quarks: First Results for Hadron Masses
}

\section{R. Frigori, ${ }^{a}$ Ch. Gattringer, ${ }^{a}$ C. B. Lang ${ }^{*},{ }^{a}$ M. Limmer, ${ }^{a}$ T. Maurer, ${ }^{b}$ D. Mohler ${ }^{a}$}

\section{and A. Schäfer ${ }^{b}$}

${ }^{a}$ Institut für Physik, FB Theoretische Physik, Karl-Franzens-Universität Graz

A-8010 Graz, Austria

${ }^{b}$ Institut für Theoretische Physik, Universität Regensburg

D-93040 Regensburg, Germany

E-mail: rafael.frigori@uni-graz.at, christof.gattringer@uni-graz.at,

christian.langeuni-graz.at markus.limmereuni-graz.at,

thilo.maureraphysik.uni-regensburg.de, daniel.mohlereuni-graz.at,

andreas.schaeferephysik.uni-regensburg.de

We present first results for a study with two mass-degenerate dynamical Chirally Improved (CI) fermions on lattices of spatial extent $2.4 \mathrm{fm}$. The CI Dirac operator obeys the Ginsparg-Wilson condition in good approximation. The pion mass we use is still large $\mathscr{O}(470 \mathrm{MeV})$ for the $16^{3} \times 32$ lattices with lattice spacing of $0.15 \mathrm{fm}$. The hadron masses are obtained with the variational technique and the results compared with earlier quenched calculations with similar lattice parameters. We find indications for the isovector, scalar meson $a_{0}(980)$ near the experimental value, in contradistinction to quenched results which always produced a mass value compatible with the first excitation $a_{0}(1450)$.

The XXV International Symposium on Lattice Field Theory

July 30 - August 42007

Regensburg, Germany

\footnotetext{
* Speaker.
} 


\section{Introduction}

It will take some time before we will be able to compute hadron properties in full QCD simulations with dynamical quarks that have full Ginsparg-Wilson $(\mathrm{GW})$ chiral symmetry. On the way to this aim we are working with the so-called Chirally Improved (CI) fermions. These are realized as truncated solutions to the GW equations for a general ansatz for the Dirac operator [1], 2]. For each site this fermion action includes several hundred neighbors with distances ranging up to three links.

Extensive quenched calculations have demonstrated good chiral as well as good scaling behavior [3]. Within the Bern-Graz-Regensburg (BGR) collaboration we have obtained results for the hadron spectrum and mesonic low energy constants in quenched simulations. These included several lattice spacings and volumes and the three light valence quarks. Emphasis in these studies has been put on deriving sophisticated techniques to analyze excited hadron states [4, [, 6] and we expect to utilize that experience for full QCD configurations eventually.

The CI Dirac operator involves many terms and as such its implementation in an HMC program is technically non-trivial. First results were obtained on $12^{3} \times 24$ lattices and presented in Ref. [17, 8]. However, the small volume (linear spatial size $1.8 \mathrm{fm}$ ) did not allow reliable results for the hadron masses.

Here we present first results for our HMC simulation on larger $16^{3} \times 32$ lattices at lattice spacing $a=0.15 \mathrm{fm}$ and for two different values of the quark masses. We use two mass degenerate light quarks, the Lüscher-Weisz gauge action, and stout smearing. One HMC-trajectory has $\mathscr{O}(100)$ steps for one unit of HMC-time. More technical details may be found in [7].

\section{Equilibration}

The approach to equilibration has been monitored by standard tests, like tracing the configuration mean plaquette or the number of conjugate gradient steps in the Monte-Carlo accept/reject step. For these two quantities we find autocorrelation lengths around 2. We skip the first 100 configurations and then analyze every fifth. Table 1 specifies the parameters for the runs discussed here.

On lattices of this size the determination of the pion mass is possible even for individual configurations. Figure 1 demonstrates the (satisfactory) time dependence of $M_{\pi}$ for every 5 th configuration of, e.g., run B.

\begin{tabular}{rrrrrrrr} 
run & $\beta_{\mathrm{LW}}$ & $c_{0}$ & $n_{\text {conf }}$ & $n_{\text {meas }}$ & $a[\mathrm{fm}]$ & $m_{\mathrm{AWI}}[\mathrm{MeV}]$ & $M_{\pi}[\mathrm{MeV}]$ \\
\hline A & 4.65 & -0.06 & 425 & 65 & $0.153(1)$ & $33(1)$ & $473(4)$ \\
B & 4.70 & -0.05 & 350 & 50 & $0.154(1)$ & $41(1)$ & $507(5)$ \\
\hline
\end{tabular}

Table 1: Parameters of the two runs discussed: run sequence, gauge coupling, bare mass parameter $c_{0}$, number of configurations $n_{\text {conf }}$, number of configurations analysed $n_{\text {meas }}$, lattice spacing $a$ assuming that the Sommer parameter $r_{0}=0.48 \mathrm{fm}$, AWI-mass, pion mass. 


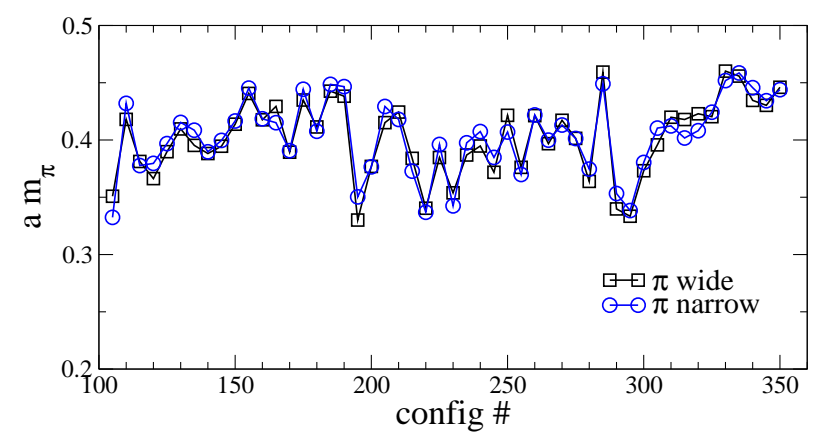

Figure 1: Time history of the pion mass determined from single configurations and pseudoscalar operator $P=\bar{d} \gamma_{5} u$ for run B. The quark smearing types used (narrow for $n n$ and wide for $w w$ ) are indicated and show excellent agreement. The fits are cosh-fits to the plateau-range $\Delta t=3-15$.
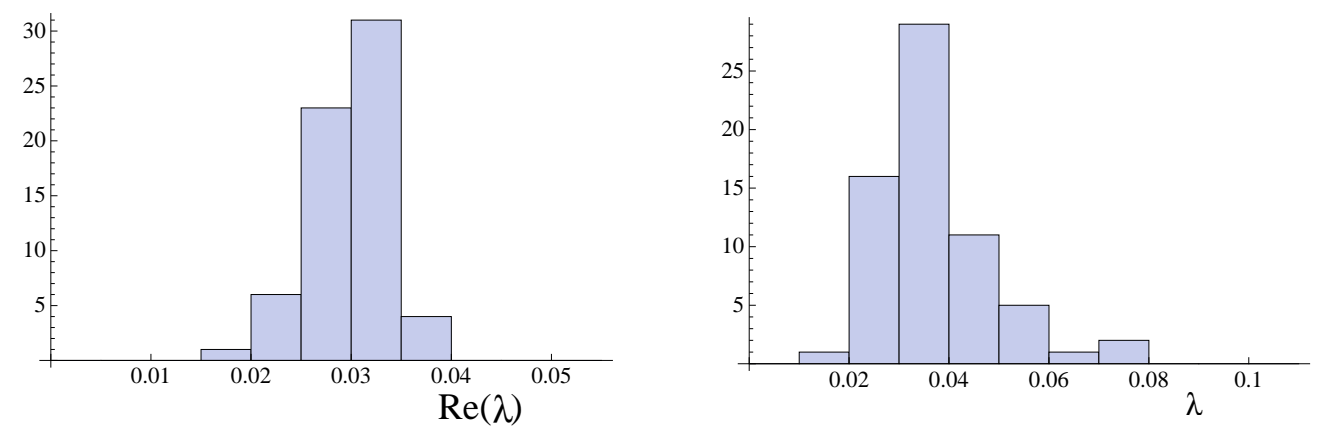

Figure 2: Histogram of the smallest values of $\operatorname{Re}(\lambda)$ (left) and real eigenvalues (right) for run A (in lattice units). The AWI-mass for this run is at $a m_{\mathrm{AWI}}=0.0264$.

We also determined the low lying eigenvalues of the CI Dirac operator (cf., [8, 9]). Figure 2 shows a histogram of the distribution of the smallest $\operatorname{Re}(\lambda)$ and of the smallest real eigenvalues. They extend inside the GW unit circle, since the CI operator is not exactly chiral.

The topological charge $v$ is determined from the number of real modes counted according to their chirality. Tunneling is a well-known problem for the GW-exact overlap fermions and sophisticated techniques have been developed to overcome this obstacle [10, 11]. Due to the diagonalization method that we used (ARPACK) we may miss a few of the modes further inside the unit GW-circle. For the CI operator we find frequent tunneling between sectors of different topological charge (cf., figure 3).

In [9] the eigenvalue distributions and properties as well as results for a hybrid approach (overlap operator and CI configurations) are discussed in more detail.

The axial Ward identity (AWI) allows one to define the renormalized quark mass through the asymptotic behavior of the ratio

$$
\frac{Z_{A}}{Z_{P}} \frac{\left\langle\partial_{t} A_{4}(\vec{p}=\overrightarrow{0}, t) X(0)\right\rangle}{\langle P(\vec{p}=\overrightarrow{0}, t) X(0)\rangle}=Z_{m} 2 m=2 m^{(r)},
$$

where $X$ is any interpolator coupling to the pion and $Z_{A}, Z_{P}$ and $Z_{m}$ denote the renormalization 

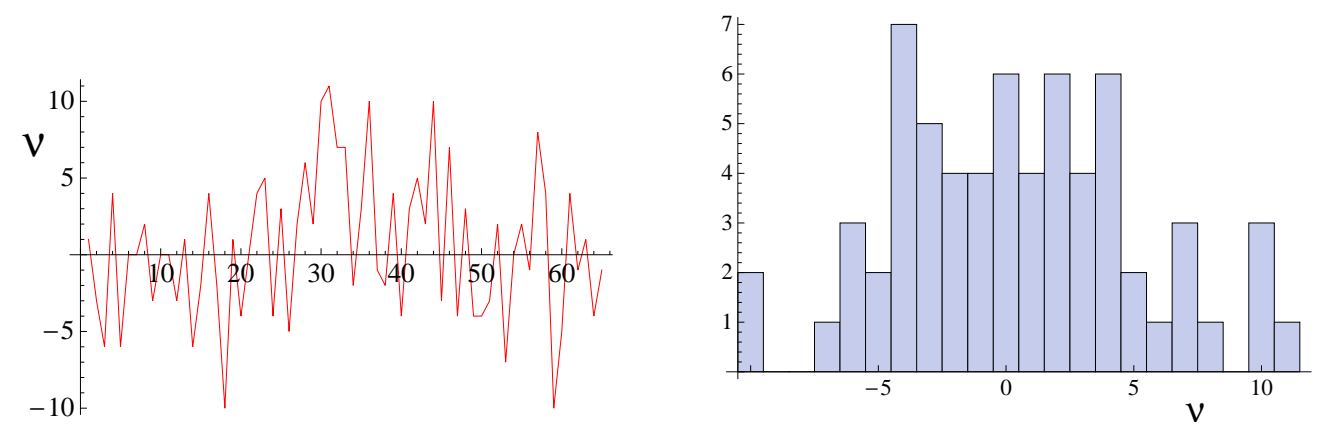

Figure 3: History (left) and histogram (right) of the topological charge $v$ during the run sequence A; 100 configurations have been discarded and then every 5 th is plotted.

factors relating to the $\overline{\mathrm{MS}}$-scheme at a scale of $2 \mathrm{GeV}$. These have been calculated for the quenched case at several values of the lattice spacing and came out close to one [12]. Determination of the values for the dynamical case is under progress. For now we work with the ratio

$$
\frac{\left\langle\partial_{t} A_{4}(\vec{p}=\overrightarrow{0}, t) P(0)\right\rangle}{\langle P(\vec{p}=\overrightarrow{0}, t) P(0)\rangle} \equiv 2 m_{\mathrm{AWI}},
$$

defining the so-called AWI-mass. The effects due to smearing of the quark sources is accounted for by comparing with point sink correlators.

In figure 4 we show the pion masses obtained for run A vs. the AWI-mass. In addition to the single point where $m_{\mathrm{val}}=m_{\text {sea }}$ we also exhibit three partially quenched data points, i.e., determined for different valence quark masses for the same dynamical sea quark configurations. We find excellent extrapolation to the chiral limit, following the leading order (GMOR) behavior. This is no surprise since we know that the leading order behavior is also found in purely quenched results.

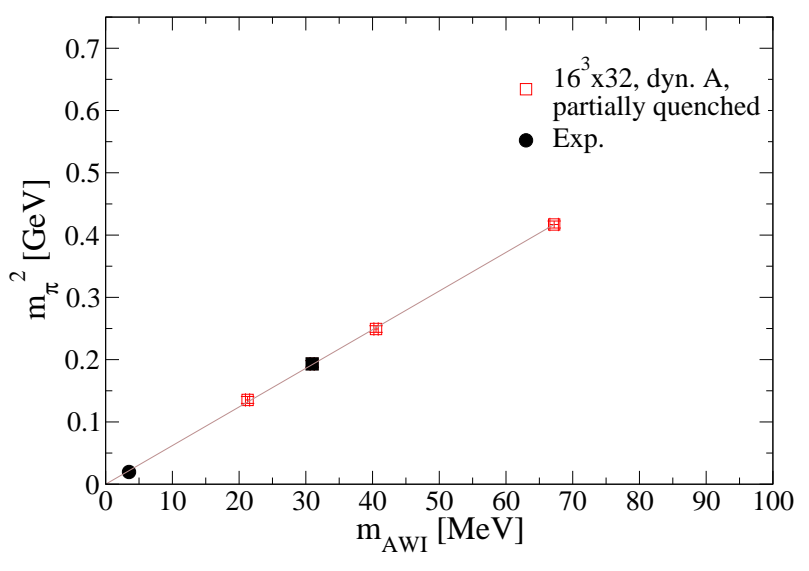

Figure 4: Pion mass squared vs. AWI-mass for run A. The data point marked by the filled square is the value where the valence quark and the sea quark masses agree, the other three points are partially quenched, i.e., the valence quark masses differ from the sea quark mass. 


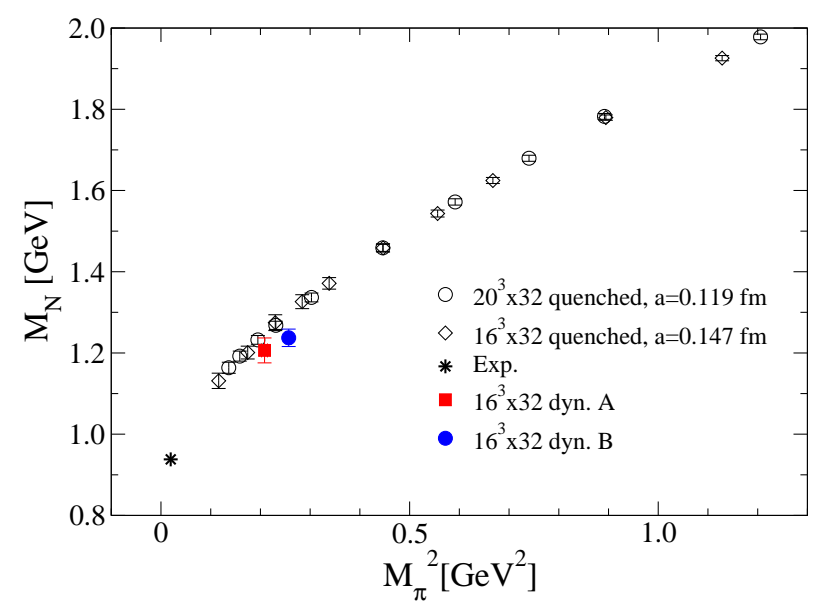

Figure 5: Results for the ground state nucleon compared to the quenched results on a lattice with similar parameters [5].

\section{Hadron masses}

For the hadron propagators we applied the techniques discussed in [4. 5. We used Jacobi smeared quark sources and the same set of interpolating operators for the hadrons. The correlation matrix between the different operators at source and sink were then analyzed invoking the so-called variational method. The presently available statistics did not allow to identify excited states and we therefore discuss here only results for the ground states.

In the quenched case [4, 5] the scale has been set with a Sommer parameter value of $r_{0}=0.5$ fm. In full QCD one usually uses somewhat smaller values. Alternative methods use $f_{\pi}, M_{\rho}$ or $M_{N}$ to set the scale. In most of our plots we exhibit the masses in units of the measured value of the nucleon mass. Only for the nucleon (and for the values of the lattice spacing in the table) we have to rely on a physical scale and there we use $r_{0}=0.48 \mathrm{fm}$.

Figure 5 compares the results for the dynamical runs with quenched results obtained on lattices with approximately the same physical size and lattice spacing [5]. In figure 6 we present a comparison for the vector meson mass and for the negative parity nucleons; for the latter we can identify the lowest two states. In all cases the dynamical results lie slightly below the quenched ones.

As a surprise came the behavior of the isovector, scalar meson $a_{0}$. In quenched calculations this state was always seen too high, compatible with the first excitation $a_{0}(1450)$. In the dynamical runs we now see the lowest mass compatible with the ground state $a_{0}(980)$ (see figure 7 ). This channel is coupled to $\pi \eta$ and we will have to study its momentum, volume and quark mass dependence for clear identification as a single particle state.

\section{Outlook}

We are currently extending our statistics for run A and plan to also produce gauge configurations at significantly smaller pion masses, for similar lattice parameters. This should allow us to 

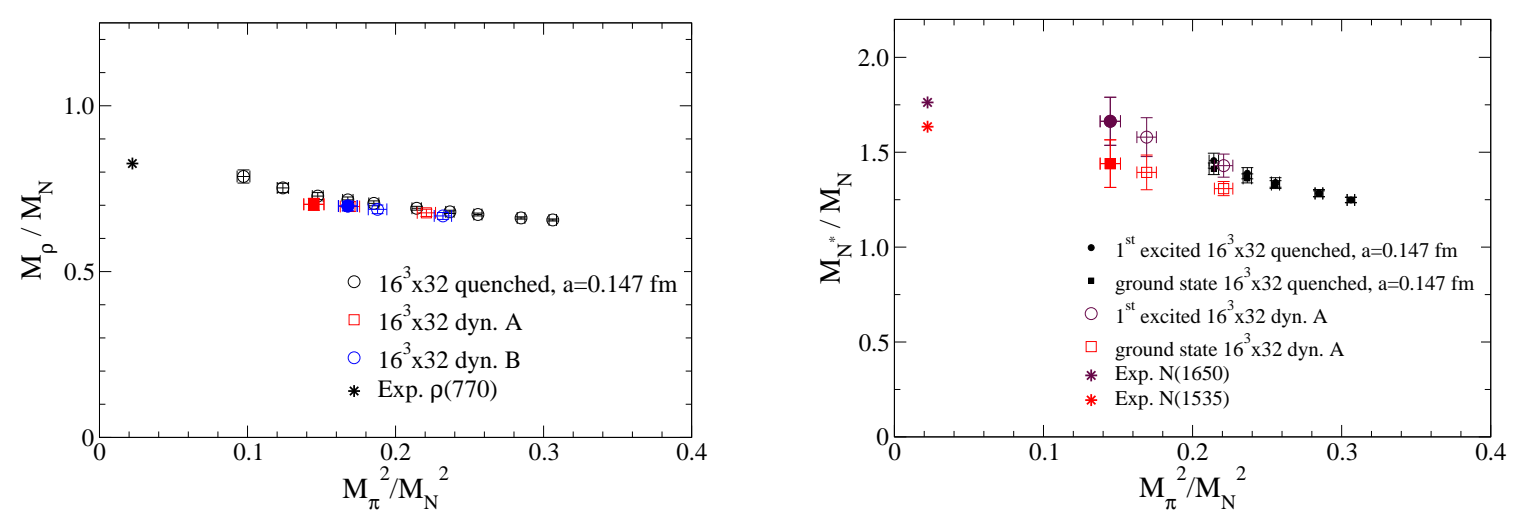

Figure 6: Left: Comparison of quenched (circles) and dynamical (squares) results for the $\rho$-meson mass in units of the measured nucleon mass. The filled squares denote fully dynamical results and the open ones partially quenched results, both for runs A and B. Right: We can identify the two lowest lying negative parity nucleons which extrapolate to the experimental values.
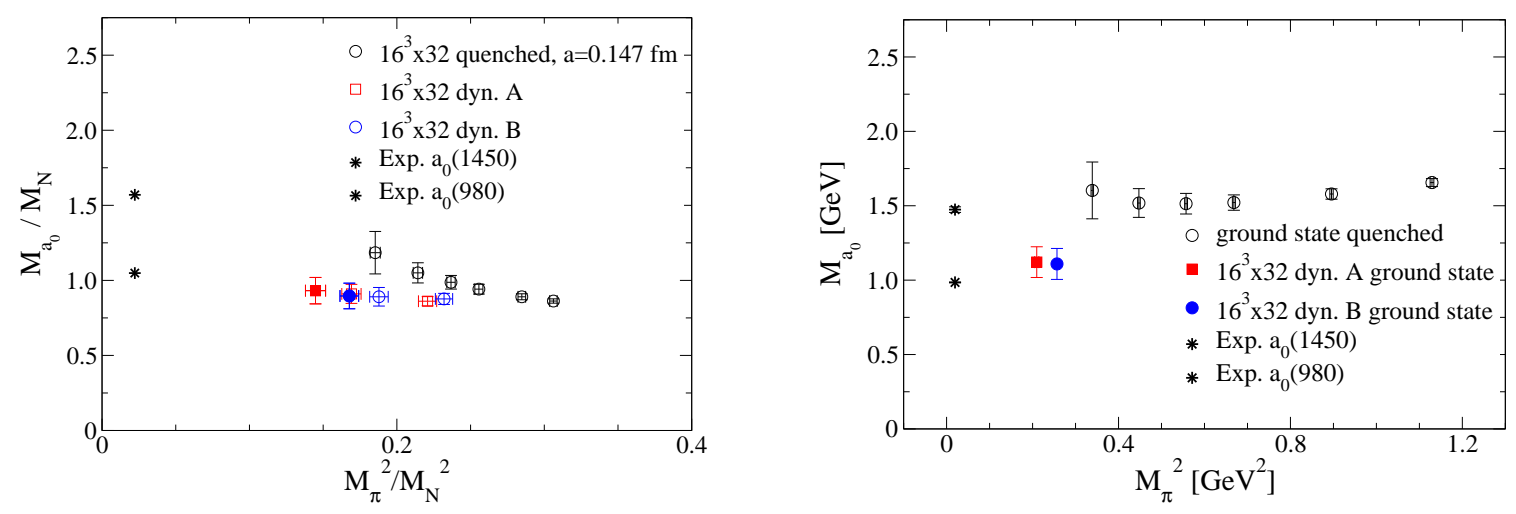

Figure 7: Contrary to the quenched runs we see in the isovector, scalar channel a low lying state compatible with the ground state $a_{0}$.

find at least the first excitations for the hadron states discussed. In particular the Roper and the Delta state are of high interest. Also the $a_{0}$ signal ought to be verified by further analysis.

\section{Acknowledgments}

The dynamical configurations have been generated on the SGI Altix 4700 of the Leibniz Rechenzentrum Munich; the hadron propagators have then been evaluated at the Boltzmann-Cluster at ZID at University of Graz. We thank both institutions for providing support. Three of the authors (R.F., D.M., M.L.) are supported by the DK W1203-N08 of the "Fonds zur Förderung der wissenschaftlichen Forschung in Österreich". A.S. and T.M. acknowledge support by DFG and BMBF. 


\section{References}

[1] C. Gattringer, A new approach to Ginsparg-Wilson fermions, Phys. Rev. D 63 (2001) 114501, [hep-lat/0003005].

[2] C. Gattringer, I. Hip, and C. B. Lang, Approximate Ginsparg-Wilson fermions: A first test, Nucl. Phys. B597 (2001) 451, hep-lat/0007042].

[3] C. Gattringer et al. [BGR Collaboration], Quenched spectroscopy with fixed-point and chirally improved fermions, Nucl. Phys. B 677 (2004) 3, hep-lat/0307013.

[4] T. Burch, C. Gattringer, L. Y. Glozman, C. Hagen, and C. Lang, Variational method for lattice spectroscopy with ghosts, Phys. Rev D73 (2006) 017502, [hep-lat/0511054].

[5] T. Burch, C. Gattringer, L. Y. Glozman, C. Hagen, D. Hierl, C. B. Lang, and A. Schäfer, Excited hadrons on the lattice: Baryons, Phys. Rev. D 74 (2006) 014504, hep-lat / 0604019.

[6] C. Gattringer, L. Y. Glozman, C. B. Lang, D. Mohler and Sasa Prevlosek, Meson spectroscopy with derivative quark sources, PoS (LATTICE2007) 123.

[7] C. B. Lang, P. Majumdar, and W. Ortner, QCD with two dynamical flavors of chirally improved quarks, Phys. Rev. D 73 (2006) 034507, [hep-lat/0512014].

[8] C. B. Lang, P. Majumdar, and W. Ortner, The condensate for two dynamical chirally improved quarks in QCD, Phys. Lett. B 649 (2007) 225, [hep-lat/0611010].

[9] C. B. Lang and M. Joergler, The eigenvalue spectrum for dynamical Chirally Improved fermions, PoS (LATTICE2007) 107.

[10] N. Cundy, S. Krieg, A. Frommer, T. Lippert, and K. Schilling, Dynamical overlap simulations using HMC, Nucl. Phys. B (Proc. Suppl.) 140 (2005) 841, hep-lat/ 0409029 .

[11] Z. Fodor, S. D. Katz, and K. K. Szabo, Dynamical overlap fermions, results with hybrid Monte-Carlo algorithm, Nucl. Phys. B (Proc. Suppl.) 140 (2005) 704, hep-lat/ 0409070 ].

[12] C. Gattringer, M. Göckeler, P. Huber, and C. B. Lang, Renormalization of bilinear quark operators for the chirally improved lattice Dirac operator, Nucl. Phys. B694 (2004) 170, hep-lat/0404006. 